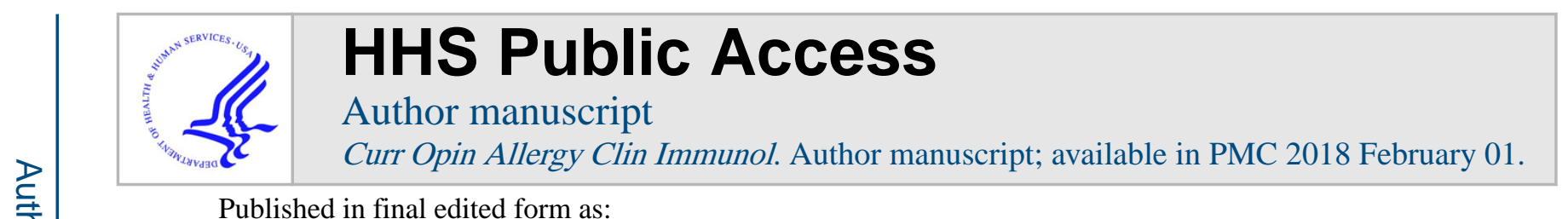

Published in final edited form as:

Curr Opin Allergy Clin Immunol. 2017 February ; 17(1): 50-54. doi:10.1097/ACI.0000000000000327.

\title{
NK cells in asthma
}

\section{Magdalena M. Gorska}

National Jewish Health, Denver, CO and University of Colorado, Aurora CO

\begin{abstract}
Purpose of review-to discuss current knowledge on natural killer (NK) cells in asthma.

Recent findings-It is now well accepted that NK cell activities go beyond cancer immune surveillance and anti-viral defense. Recent reports indicate that NK cells are activated in response to allergens in vivo. NK cells promote allergic sensitization, type- 2 immune response, development of eosinophilic inflammation and airway hyperresponsiveness. NK cells are activated by RSV and other respiratory viruses. When infection occurs in the setting of active allergic inflammation, NK cells augment its magnitude and contribute to asthma exacerbations. Proasthma activities of NK cells can be programmed during embryogenesis through maternal exposure to environmental pollutants. Prenatally-programmed NK cells produce type- 2 and type- 3 cytokines and mediate asthma predisposition. NK cells can also act as asthma antagonists. NK cells contribute to the resolution of inflammation through suppression of antigen-specific CD4+ T cells and type- 3 immunity. When viral infection occurs in naïve mice prior to allergic sensitization, NK cells antagonize type- 2 immunity and prevent development of asthma.
\end{abstract}

Summary-NK cells are non-redundant participants of allergic inflammation. The environmental context determines whether NK cells act as protagonists or antagonists.

\section{Keywords}

natural killer cells; type-2 immunity; asthma; mouse models

\section{Introduction}

Allergic asthma arises as result of a complex interplay between stromal cells of the lung and cells of innate and adaptive immunity. Innate immunity contributes to initiation, maintenance and resolution of the allergic response. Some innate immune cells play a master regulatory role and some exert specific effector functions. Innate lymphoid cells belong to master regulators of allergic inflammation. The importance of innate lymphoid type- 2 cells (ILC2s) has been highlighted in multiple original research and review articles. Less is known about other innate lymphoid cells, including NK cells. The purpose of this review is to provide a summary of current knowledge on the role of NK cells in asthma.

Correspondence to Magdalena M. Gorska, National Jewish Health, Department of Medicine, Division of Allergy and Clinical Immunology, 1400 Jackson St, Denver, CO 80206, Phone: 303-398-1656, Fax: 303-298-1806, gorskam@ njhealth.org. 


\section{NK cells in human asthma}

There are several reports on NK cells in human asthma. Early studies demonstrated increased cytotoxic activity of NK cells in peripheral blood from asthmatic subjects [1,2]. This activity decreased immediately after allergen challenge suggesting either migration of NK cells from circulation to the lung, or suppression of NK cell cytotoxicity by an allergen or allergic inflammatory milieu. A more recent study by Lin et al. compared asthmatic children during acute exacerbation and children with stable asthma [3]. The former group had higher numbers of NK cells in the blood. Barnig et al. investigated adult patients and found that NK cells from severe asthmatics expressed significantly higher levels of the activating receptor NKG2D and the activation marker CD69 relative to cells from mild asthmatics and healthy subjects [4]. In severe asthmatics there was a positive correlation between NK cell number and peripheral blood eosinophil count. In addition, the eosinophil number had a positive relationship with NK cell surface expression of CD69 and NKG2D. To determine the impact of NK cells on eosinophils, the authors performed co-culture experiments. They found that NK cells triggered apoptosis of eosinophils. The mechanism might have involved the pro-resolving lipid lipoxin A4. Lipoxin A4-exposed NK cells induced more apoptotic eosinophils compared to unexposed cells. The NK cell-mediated apoptosis of eosinophils is an exciting finding on its own merit but it does not explain the positive correlation between NK cell and eosinophil numbers. Wei et al. studied cytokine profiles of NK cells in asthma [5]. Healthy subjects served as controls. Patients with asthma had increased frequencies of IL-4-producing NK cells and decreased frequencies of IFN $\gamma$ producing NK cells in the blood. NK cell clones were then obtained by limiting dilution. Clones from asthmatic subjects had higher levels of transcripts for IL-4 and IL-6 and lower levels of transcripts for IFN $\gamma, \mathrm{TNFa}$ and lymphotoxin. The relationship between numbers of IL-4-producing NK cells and eosinophils was not determined.

\section{NK cells in mouse models of asthma}

Mouse models have shown that a function of NK cells in asthma can be protagonistic or antagonistic, dependent on the environmental agent that is used to elicit the disease (allergen, diesel exhaust particles and virus) and the phase of the disease (sensitization, the effector phase and the resolution phase).

NK cells act as protagonists of allergic sensitization in allergen-induced models of asthma. Using the ovalbumin (OVA)-induced model of asthma Korsgren et al. and Mathias et al. showed that depletion of NK cells either prior to immunization (depleting antibodies) or throughout the life of an animal (genetically-modified mice) prevents production of IL-4 in lymphoid organs, generation of ovalbumin-specific IgE, infiltration of lungs by eosinophils and $T$ cells and production of pulmonary type- 2 cytokines $[6,7]$. Thus, NK cells are likely necessary for differentiation of naïve T cells into Th2 cells and initiation of adaptive type- 2 responses. Mathias and colleagues investigated importance of NK cells in T cell priming in an adoptive transfer model [7]. CD4+ T cells from sensitized NK cell-deficient mice were unable to induce eosinophilia in lungs of allergen-challenged RAG1-/- recipients, while CD4+ T cells from WT recipients were fully capable to do so. The mechanism of $\mathrm{T}$ cell priming might have involved dendritic cells. Depletion of NK cells reduced a number of 
$\mathrm{CD} 11 \mathrm{c}+$ cell in the spleen and the lung. NK cells are recognized regulators of dendritic cells. NK cells can induce differentiation of monocytes into dendritic cells via secretion of GMCSF, attract dendritic cells via CCL3, CCL4 and CCL5 and mature them via TNFaand IFN $\gamma[8-11]$.

In addition to positive effects on allergic sensitization, NK cells promote the initiation of asthma effector mechanisms. Ple and colleagues observed that the onset of the effector phase was characterized by expansion of NK cells in mediastinal lymph nodes [12]. Lymph node NK cells expressed higher levels of CD86, suggesting interactions of NK cells with CD4+ T cells. To understand a significance of these observations, Ple at al., administered NK celldepleting antibodies prior to the effector phase. NK cell depletion resulted in a sharp decrease of eosinophil numbers in the lung.

A manuscript by Farhadi et al. provided insight into mechanisms of NK cell activation by allergens [13]. The investigators started their story by demonstrating that that airway inflammation in the house dust mite (HDM)-induced model of asthma was dependent on NKG2D. NKG2D is an activating receptor expressed on all NK cells and subsets of T cells [14]. It serves as a major receptor for recognition and elimination of unhealthy/stressed cells including infected and cancer cells as well as cells damaged by environmental toxins. The ligands of NKG2D are self-proteins that are not expressed on healthy cells but become upregulated by cellular stress. Farhadi and colleagues demonstrated induction of the NKG2D ligand MULT-1 in the lung of HDM-exposed mice. NKG2D deficiency (KO mice) reduced numbers of NK cells, T cells and eosinophils in the HDM-challenged lung, prevented production of IL- 4 and IL- 13 by pulmonary CD4+ T cells and blocked generation of total and HDM-specific IgE. Adoptive transfer of WT NK cells restored allergic inflammatory responses in NKG2D KO recipients. These responses were not restored upon a transfer of NKG2D-deficient NK cells, granzyme B-deficient NK cells or WT T cells. In summary, the manuscript indicated a critical role of NKG2D and granzyme B in NK cellmediated activation of type- 2 immunity in asthma.

Studies by Korsgen, Mathias, Ple and Farhadi have shown that NK cells are participants of inflammation in asthma. We have postulated that NK cells may also act as carriers of asthma predisposition. We have focused on asthma predisposition that is acquired through prenatal exposure to diesel exhaust [15]. In our model, female mice were repeatedly exposed to diesel exhaust particles (DEP) during pregnancy. Offspring underwent immunization and challenge with OVA. The immunization strategy differed from this used in traditional models. It was administered very early in life (the neonatal period) and therefore was "suboptimal". Consequently, the described treatment with OVA did not elicit inflammation in normal pups (pups born to unexposed mothers). The inflammation developed only in predisposed pups i.e. pups born to DEP-exposed mothers. In this model, NK cells acquired an unusual phenotype - they produced type- 2 cytokines (IL-5 and IL-13) and IL-17A. In fact, NK cells constituted the majority (>50\%) of IL-5/IL-13/IL-17-producing pulmonary leukocytes. Through production of these cytokines, NK cells were likely to have a direct influence on eosinophils, bronchial epithelium and airway hyperresponsiveness. We postulated that the type-2/3 cytokine expression profile of NK cells was a result of environmentally-induced reprogramming of fetal NK precursors. One potential mechanism 
of this reprogramming may involve activation of the transcriptional factor aryl hydrocarbon receptor (AhR). AhR is a known target for DEP-derived polycyclic aromatic hydrocarbons [16]. AhR is an established regulator of the IL-17A gene transcription [17, 18]. Some reports suggest the positive role of AhR in IL-5 and IL-13 production [19, 20]. Thus, AhR may provide a molecular link between maternal exposure to DEP and cytokine production in offspring NK cells. In fact, we observed increased activation of AhR in prenatally-exposed pups. The reprogrammed NK cells mediated asthma predisposition. Depletion of these cells prevented development of asthma.

NK cells were also suggested to mediate virally-induced asthma exacerbations. To elicit asthma exacerbation, Lunding at al. injected poly(inosinic-cytidylic) acid [poly(I-C)] to mice with an established allergic inflammation of airways (the end of the challenge phase in the OVA model) [21]. Poly(I-C) is a viral "mimic" that signals through Toll-like receptor 3 (TLR3). Injection of poly(I-C) exacerbated asthma and increased numbers of IL-17Aproducing NK cells in the lung. Depletion of NK cells one day before the poly(I-C) injection reduced levels of IL-17A and prevented asthma exacerbation. Asthma exacerbation was also prevented by depletion of IL-17A. The manuscript suggests a critical role of IL-17Aproducing NK cells in the pathogenesis of virally-triggered asthma exacerbations.

In the model presented by Lunding et al. NK cell activation by a viral mimic occurred during an ongoing allergic response. In these settings, NK cells acted as enhancers of asthma.

Manuscript by Kaiko et al. shows a different scenario; when viral infection occurs in a naïve mouse, in the absence of an allergic inflammatory milieu, NK cells acquire anti-asthma capacities that are attributable to the production of IFN $\gamma$ [22]. Depletion of NK cells at the time of infection with respiratory syncytial virus (RSV) resulted in the reduction of IFN $\gamma$ synthesis, augmentation of virus-specific Th2 responses, increased mucus production and eosinophilia in the lung. Th2 responses persisted over long term; they were detectable at day 42 post infection. Administration of IFN $\gamma$ to NK cell-depleted mice on day 4 post infection reversed the cytokine pattern. To study the role of virally-primed NK cells on subsequent responses to allergens, Kaiko et al. depleted NK cells in RSV-infected mice and then subjected these mice to a series of treatments with aerosolized OVA. In the absence of an adjuvant or other immune stimulator, mucosal exposure to OVA is known to induce tolerance to this allergen. NK cell depletion during RSV infection broke this tolerance, increased type- 2 cytokine production in lymph nodes and eosinophil numbers in the lung. The authors suggest that NK cell deficiency in pediatric patients may provide a mechanistic link between severe RSV infection and a risk of childhood asthma.

Haworth et al. investigated a role of NK cells in the resolution of allergic inflammation [23]. The investigators used an OVA model of experimental asthma. The NK cell depleting antibody was administered 4 days after initial challenge with OVA i.e. during the effector phase of allergic response. Mice were examined during the resolution phase. In these settings, depletion of NK cells prevented the resolution of peribronchial cellular infiltrates (histology). Interestingly, NK cell depletion had no effect on pulmonary IL-5 and IL-13 and only marginal effect on eosinophils. Instead, depletion of NK cells led to significant increase in the number of OVA-specific CD4+ T cells in the lung and increased levels of pulmonary IL-23, IL-17A and IFN $\gamma$. Therefore, the role of NK cells during the resolution phase is 
likely to suppress antigen-specific Th17 and Th1 immunity. This is reminiscent of reports on the critical role of NK cells in suppression of myelin-specific Th17 cells in experimental autoimmune encephalomyelitis (EAE) [24, 25]. In allergic inflammation, the suppressive functions of NK cells may be driven by resolvin E1, as depletion of NK cells interfered with the ability of exogenous resolvin E1 to limit inflammation [23].

Figure 1 summarizes the proposed activation mechanisms and functions of NK cells in asthma.

\section{Conclusion}

The manuscripts described above show that NK cells may promote or inhibit type-1, type-2 and type-3 immunity. The outcome of NK cell activation is likely dependent on many factors including the type of the environmental trigger, the type and the phase of inflammation, the cytokine/mediator milieu, the cellular partners, the developmental stage of the organism and the differentiation stage of the NK cell. In this regard, NK cells are not different from other cell types of the immune response. Similar divergent and often opposing functions have been reported for T cells, dendritic cells and many other cells. One possible explanation for this is that immune cells never work in isolation; instead they act as components of large networks; in these networks, functions of an individual node are more influenced by a network than by intrinsic properties of a node.

The presented manuscripts raise lots of enthusiasm and strongly suggest the importance of NK cells in asthma. However these studies are not without caveats. The main limitations are less-than-perfect approaches used to deplete NK cells. The majority of the manuscripts used depleting antibodies, either anti-NK1.1 or anti-asialo-GM1. The use of these antibodies has been a method-of-choice in NK cell research for many years. However, these antibodies are not $100 \%$ specific as they eliminate some non-NK cells including NKT cells (anti-NK1.1) and subsets of activated T cells (anti-asialo-GM1). One group utilized transgenic mice that overexpressed the inhibitory receptor Ly49A under control of the mouse granzyme A genomic sequence [7]. The mice had severely reduced numbers of NK cells. The caveat was that Ly49A was also expressed on 50\% of T cells [26]. Therefore, a phenotype of transgenic mice might be also attributable to the inhibition of $\mathrm{T}$ cells by Ly49A.

In summary, we made several very promising observations on NK cells in asthma. Further studies are needed to confirm current findings and elucidate immunological and molecular mechanisms of NK cell priming, activation and effector functions in this disease.

\section{Acknowledgments}

None

Financial support and sponsorship

This work was supported by the National Institutes of Health grant 1R01HL122995 


\section{References}

1. Timonen T, Stenius-Aarniala B. Natural killer cell activity in asthma. Clin Exp Immunol. 1985; 59:85-90. [PubMed: 3971600]

2. Jira M, Antosova E, Vondra V, et al. Natural killer and interleukin-2 induced cytotoxicity in asthmatics. I. Effect of acute antigen-specific challenge. Allergy. 1988; 43:294-298. [PubMed: 2455455]

3. Lin SJ, Chang LY, Yan DC, et al. Decreased intercellular adhesion molecule-1 (CD54) and Lselectin (CD62L) expression on peripheral blood natural killer cells in asthmatic children with acute exacerbation. Allergy. 2003; 58:67-71. [PubMed: 12580810]

4. Barnig C, Cernadas M, Dutile S, et al. Lipoxin A4 regulates natural killer cell and type 2 innate lymphoid cell activation in asthma. Sci Transl Med. 2013; 5:174ra26.

5. Wei H, Zhang J, Xiao W, et al. Involvement of human natural killer cells in asthma pathogenesis: natural killer 2 cells in type 2 cytokine predominance. J Allergy Clin Immunol. 2005; 115:841-847. [PubMed: 15806008]

6. Korsgren M, Persson CG, Sundler F, et al. Natural killer cells determine development of allergeninduced eosinophilic airway inflammation in mice. J Exp Med. 1999; 189:553-562. [PubMed: 9927517]

7*. Mathias CB, Guernsey LA, Zammit D, et al. Pro-inflammatory role of natural killer cells in the development of allergic airway disease. Clin Exp Allergy. 2014; 44:589-601. This is a recent manuscript that confirms previous reports on protagonistic effects of NK cells on allergic sensitization, type-2 immunity and asthma. [PubMed: 24397722]

8. Zhang AL, Colmenero P, Purath U, et al. Natural killer cells trigger differentiation of monocytes into dendritic cells. Blood. 2007; 110:2484-2493. [PubMed: 17626840]

9. Eberlein J, Nguyen TT, Victorino F, et al. Comprehensive assessment of chemokine expression profiles by flow cytometry. J Clin Invest. 2010; 120:907-923. [PubMed: 20197626]

10. Piccioli D, Sbrana S, Melandri E, Valiante NM. Contact-dependent stimulation and inhibition of dendritic cells by natural killer cells. J Exp Med. 2002; 195:335-341. [PubMed: 11828008]

11. Gerosa F, Baldani-Guerra B, Nisii C, et al. Reciprocal activating interaction between natural killer cells and dendritic cells. J Exp Med. 2002; 195:327-333. [PubMed: 11828007]

12. Ple C, Barrier M, Amniai L, et al. Natural killer cells accumulate in lung-draining lymph nodes and regulate airway eosinophilia in a murine model of asthma. Scand J Immunol. 2010; 72:118-127. [PubMed: 20618770]

13*. Farhadi N, Lambert L, Triulzi C, et al. Natural killer cell NKG2D and granzyme B are critical for allergic pulmonary inflammation. J Allergy Clin Immunol. 2014; 133:827-35 e3. The manuscript describes a novel mechanism of NK cell activation in asthma. The mechanism involves the "stress-surveillance" receptor NKG2D. [PubMed: 24290277]

14. Lanier LL. NKG2D Receptor and Its Ligands in Host Defense. Cancer Immunol Res. 2015; 3:575582. [PubMed: 26041808]

15*. Manners S, Alam R, Schwartz DA, Gorska MM. A mouse model links asthma susceptibility to prenatal exposure to diesel exhaust. J Allergy Clin Immunol. 2014; 134:63-72. This is the first report that describes a pivotal role of NK cells in transmission of asthma predisposition across generations. [PubMed: 24365139]

16. Stevens EA, Mezrich JD, Bradfield CA. The aryl hydrocarbon receptor: a perspective on potential roles in the immune system. Immunology. 2009; 127:299-311. [PubMed: 19538249]

17. Veldhoen M, Hirota K, Westendorf AM, et al. The aryl hydrocarbon receptor links TH17-cellmediated autoimmunity to environmental toxins. Nature. 2008; 453:106-109. [PubMed: 18362914]

18. Veldhoen M, Hirota K, Christensen J, et al. Natural agonists for aryl hydrocarbon receptor in culture medium are essential for optimal differentiation of Th17 T cells. J Exp Med. 2009; 206:4349. [PubMed: 19114668]

19. Sibilano R, Frossi B, Calvaruso M, et al. The aryl hydrocarbon receptor modulates acute and late mast cell responses. J Immunol. 2012; 189:120-127. [PubMed: 22649193] 
20. Zhou Y, Tung HY, Tsai YM, et al. Aryl hydrocarbon receptor controls murine mast cell homeostasis. Blood. 2013; 121:3195-3204. [PubMed: 23462117]

$21 *$. Lunding LP, Webering S, Vock C, et al. Poly(inosinic-cytidylic) acid-triggered exacerbation of experimental asthma depends on IL-17A produced by NK cells. J Immunol. 2015; 194:56155625. This manuscript provides evidence for an essential role of NK cells in asthma exacerbations. [PubMed: 25972482]

22. Kaiko GE, Phipps S, Angkasekwinai P, et al. NK cell deficiency predisposes to viral-induced Th2type allergic inflammation via epithelial-derived IL-25. J Immunol. 2010; 185:4681-4690. [PubMed: 20855881]

23. Haworth O, Cernadas M, Levy BD. NK cells are effectors for resolvin E1 in the timely resolution of allergic airway inflammation. J Immunol. 2011; 186:6129-6135. [PubMed: 21515793]

24. Hao J, Liu R, Piao W, et al. Central nervous system (CNS)-resident natural killer cells suppress Th17 responses and CNS autoimmune pathology. J Exp Med. 2010; 207:1907-1921. [PubMed: 20696699]

25. Hao J, Campagnolo D, Liu R, et al. Interleukin-2/interleukin-2 antibody therapy induces target organ natural killer cells that inhibit central nervous system inflammation. Ann Neurol. 2011; 69:721-734. [PubMed: 21425186]

26. Kim S, Iizuka K, Aguila HL, et al. In vivo natural killer cell activities revealed by natural killer cell-deficient mice. Proc Natl Acad Sci U S A. 2000; 97:2731-2736. [PubMed: 10694580] 


\section{Key points}

- $\quad$ NK cells are activated in human asthma

- $\quad$ NK cells play a critical role in allergic sensitization and induction of the effector phase in allergen-induced mouse models of asthma.

- Prenatal exposures to environmental pollutants lead to the emergence of type-2/3 NK cells that mediate predisposition to asthma.

- Virally-activated NK cells augment pre-existing allergic inflammation and contribute to asthma exacerbations. When infection occurs in naïve animals prior to allergic sensitization, NK cells prevent type- 2 responses and asthma.

- $\quad$ NK cells can be activated by pro-resolving lipid mediators and contribute to resolution of allergic inflammation. 


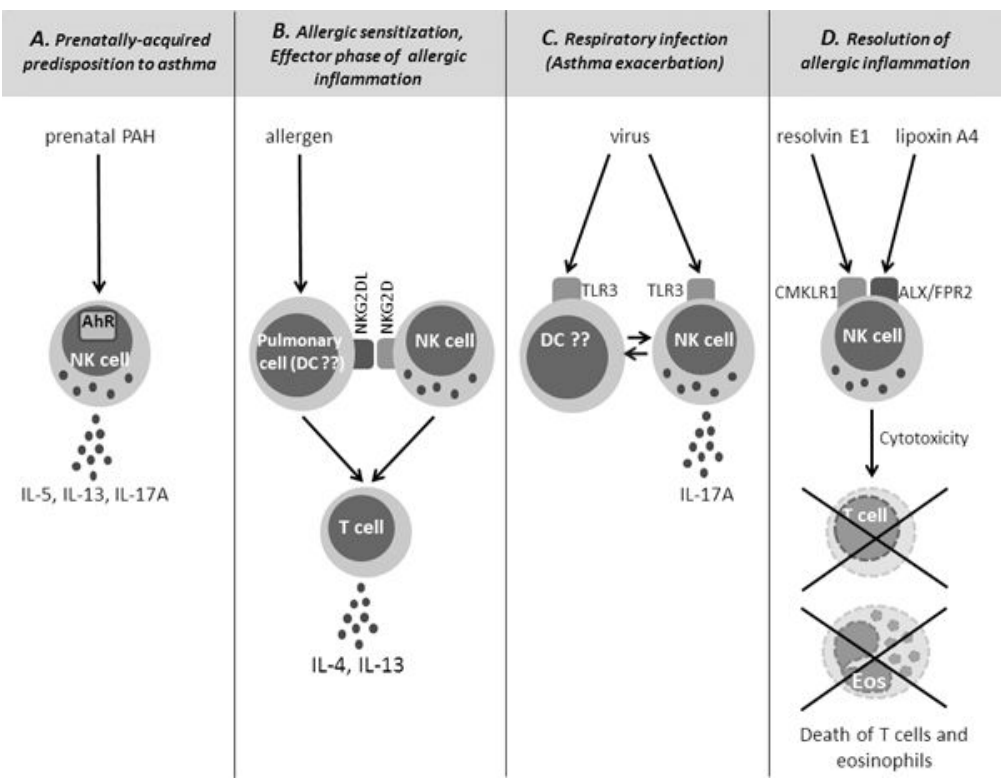

Figure 1.

Proposed activation mechanisms and functions of NK cells in asthma.

A. NK cells are activated in utero as a result of maternal exposure to polycyclic aromatic hydrocarbons (PAH). PAH go through the placenta and activate fetal NK cell precursors through the transcriptional factor aryl hydrocarbon receptor (AhR). Prenatally-primed NK cells produce IL-5, IL-13 and IL-17A and mediate predisposition to asthma. B. Allergens activate NK cells through induction of ligands for the NK cell receptor NKG2D on the surface of pulmonary cells (e.g. dendritic cells (DC)). NKG2DL recognition by NK cells is required for $\mathrm{CD} 4 \mathrm{~T}$ cell differentiation into Th2 cells and allergic sensitization. NK cells also play a role in CD4 $\mathrm{T}$ cell activation during the effector phase of allergic inflammation. C. Viruses activate NK cells through Toll-like receptors (TLR), including TLR3. The activation process may be direct or indirect (through dendritic cells). Virally-activated NK cells produce IL-17A and contribute to asthma exacerbations. D. NK cells are also activated through the engagement of the Chemokine-like Receptor 1 (CMKLR1) and the Lipoxin A4 Receptor/Formyl Peptide Receptor 2 (ALX/FPR2) by pro-resolving lipid mediators resolvin E1 and lipoxin A4, respectively. Lipid-activated NK cells kill T cells and eosinophils (Eos) and mediate resolution of allergic inflammation. 